Document downloaded from:

http://hdl.handle.net/10251/96944

This paper must be cited as:

Gutierrez Garcia, J.; Rodríguez López, J.; Romaguera Bonilla, S.; Sanchis, M. (2016). The Wijsman topology of a fuzzy metric space. Fuzzy Sets and Systems. 300:57-71. doi:10.1016/j.fss.2015.08.005

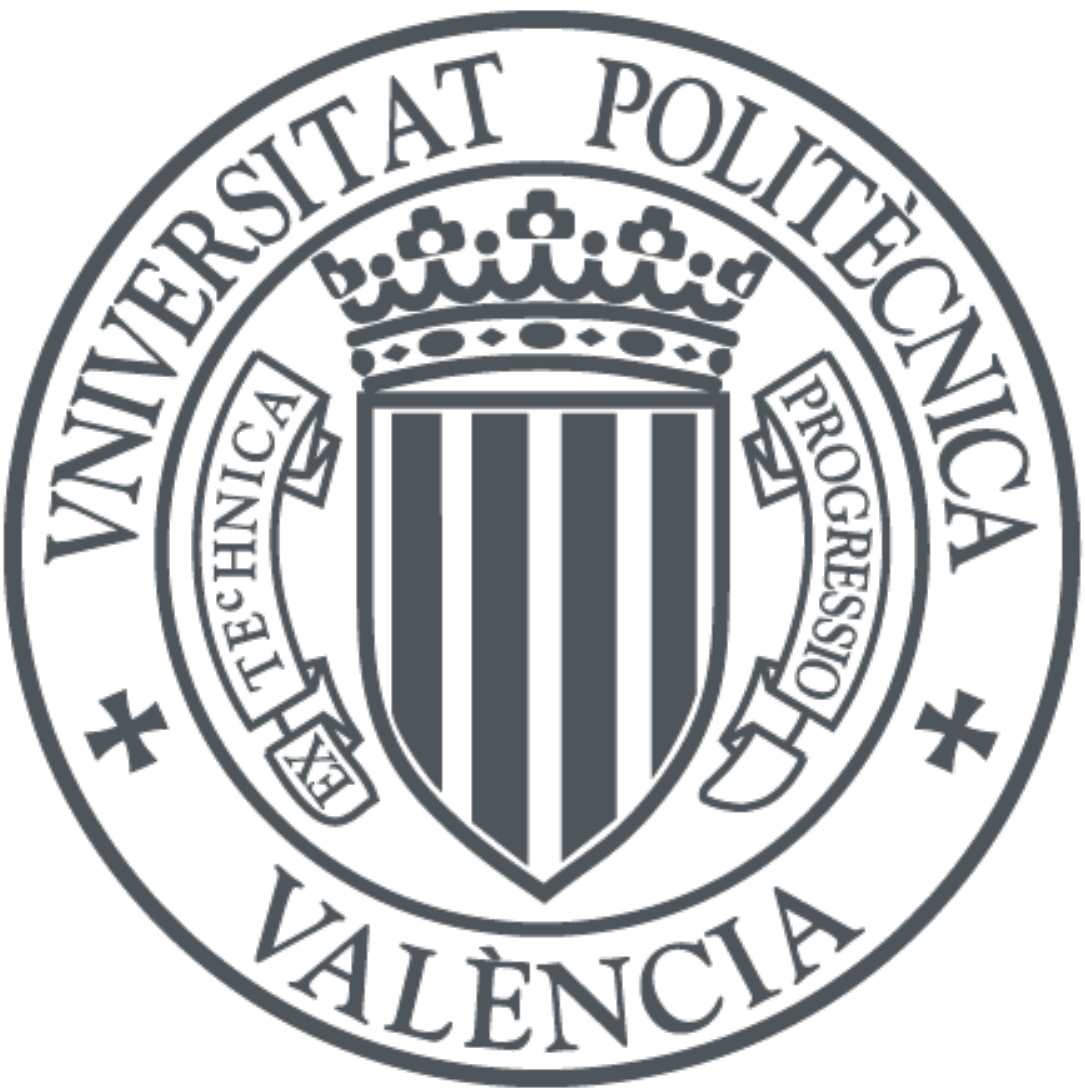

The final publication is available at

https://doi.org/10.1016/j.fss.2015.08.005

Copyright Elsevier

Additional Information 


\title{
The Wijsman topology of a fuzzy metric space
}

\author{
J. Gutiérrez García ${ }^{1)}$, J. Rodríguez-López ${ }^{2)}$, S. Romagueraa), M. Sanchis ${ }^{3)}$ \\ 1) Departamento de Matemáticas, \\ Universidad del País Vasco UPV/EHU, Apdo. 644, 48080 Bilbao, Spain \\ E-mail: javier.gutierrezgarcia@ehu.eus \\ 2) Instituto Universitario de Matemática Pura y Aplicada, \\ Universitat Politècnica de València, 46022 Valencia, Spain \\ E-mail: jrlopez@mat.upv.es, sromague@mat.upv.es \\ ${ }^{3)}$ Institut Universitari de Matemàtiques i Aplicacions de Castelló(IMAC), \\ Universitat Jaume I, Campus del Riu Sec. s/n, 12071 Castelló, Spain \\ E-mail: sanchis@mat.uji.es
}

\begin{abstract}
We introduce and study the notions of lower Wijsman topology, upper Wijsman topology and Wijsman topology of a fuzzy metric space in the sense of Kramosil and Michalek. In particular, quasi-uniformizability, uniformizability, quasi-metrizability and metrizability of these topologies are discussed. Their relations with other hypertopologies are also analyzed. Corresponding results to the Wijsman topology of a metric space are deduced from our approach with the help of the standard fuzzy metric.
\end{abstract}

Key words: Fuzzy metric space, Wijsman topology, bispace, separable, second countable, (quasi-)metrizable, the Hausdorff fuzzy metric, Vietoris topology.

\section{Introduction and preliminaries}

Wijsman introduced in [30] a kind of convergence for sequences of subsets of $\mathbb{R}^{n}$ which is suitable for working with unbounded sets. Wijsman convergence motivated the introduction and deep study of a topology on the set $\mathcal{C}_{0}(X)$ of all nonempty closed subsets of a metric space $(X, d)$, the so-called Wijsman topology (see e.g. $[1,2]$ and their references, $[10,12,25,31]$, and $[6,5]$ for more recent contributions). In particular, the Wijsman topology of a metric space $(X, d)$ is weaker than the topology of the Hausdorff distance of $(X, d)$.

Since, on the one hand, Wijsman convergence is considered by many mathematicians as the point of departure for the modern theory of set convergence ([1, Section 2.1, p. 34], [2, Section 1]) and, on the other hand, there exists a well-established theory of the Hausdorff fuzzy metric for fuzzy metric spaces, the problem of extending the notion of Wijsman topology to a fuzzy metric space $(X, M, *)$ and investigate, among other properties, its relation with the topology induced by the Hausdorff fuzzy metric of $(X, M, *)$, arises in a natural way. In Section 2 we explore this problem and show that the situation presents some interesting differences with respect to the classical case of metric spaces. We also establish some fundamental results on uniformizability and 
metrizability of the Wijsman topology of a fuzzy metric space from which we deduce as a consequence the classical results corresponding to metric spaces with the help of the standard fuzzy metric of a metric space.

Furthermore, the Wijsman topology has been considered as the building blocks of the lattice of hypertopologies because several hypertopologies can be obtained by taking supremum or infimum of Wijsman topologies (see $[3,11,12]$ ). Then, it is natural to wonder if this also occurs in the fuzzy context. Section 3 will be devoted to start this study.

In the sequel the letters $\mathbb{R}$ and $\mathbb{N}$ will denote the set of real numbers and the set of positive integer numbers, respectively.

Our basic references for quasi-metric spaces and quasi-uniform spaces are [9] and [15], and for general topology it is [14].

Let us recall that a quasi-uniformity on a set $X$ is a filter $\mathcal{U}$ on $X \times X$ such that:

(QU1)) for each $U \in \mathcal{U}, \Delta \subseteq U$, where $\Delta=\{(x, x): x \in X\}$;

(QU2) for each $U \in \mathcal{U}$ there is $V \in \mathcal{U}$ such that $V^{2} \subseteq U$, where $V^{2}=\{(x, y) \in X \times X$ : there is $z \in X$ with $(x, z) \in V$ and $(z, y) \in V\}$.

If, in addition, $\mathcal{U}$ satisfies:

(QU3) for each $U \in \mathcal{U}, U^{-1} \in \mathcal{U}$, where $U^{-1}=\{(x, y) \in X \times X:(y, x) \in U\}$,

then $\mathcal{U}$ is called a uniformity (on $X$ ).

By a (quasi-) uniform space we mean a pair $(X, \mathcal{U})$ such that $X$ is a set and $\mathcal{U}$ is a (quasi-)uniformity on $X$.

Given a quasi-uniformity $\mathcal{U}$ on a set $X$, the filter $\mathcal{U}^{-1}$ defined on $X \times X$ by $\mathcal{U}^{-1}=$ $\left\{U^{-1}: U \in \mathcal{U}\right\}$ is also a quasi-uniformity on $X$, called the conjugate of $\mathcal{U}$, and the filter $\mathcal{U}^{s}=\mathcal{U} \vee \mathcal{U}^{-1}$ is a uniformity on $X$. Obviously each uniformity $\mathcal{U}$ is a quasi-uniformity where $\mathcal{U}=\mathcal{U}^{-1}$.

Each quasi-uniformity $\mathcal{U}$ on $X$ induces a topology $\tau_{\mathcal{U}}$ on $X$ such that a neighborhood base for each point $x \in X$ is given by $\{U(x): U \in \mathcal{U}\}$, where $U(x)=\{y \in X:(x, y) \in$ $\mathcal{U}\}$.

A bitopological space (or simply, a bispace) is a triple $\left(X, \tau_{1}, \tau_{2}\right)$ such that $X$ is a set, and $\tau_{1}$ and $\tau_{2}$ are topologies on $X$.

A bispace $\left(X, \tau_{1}, \tau_{2}\right)$ is called quasi-uniformizable if there is a quasi-uniformity $\mathcal{U}$ on $X$ such that $\tau_{\mathcal{U}}=\tau_{1}$ and $\tau_{\mathcal{U}^{-1}}=\tau_{2}$. If $\tau_{1}$ is a $T_{0^{-}}$-topology, we say that $\left(X, \tau_{1}, \tau_{2}\right)$ is a quasi-uniformizable $T_{0}$-bispace. In this case $\tau_{2}$ is also a $T_{0}$ topology and $\left(X, \tau_{1} \vee \tau_{2}\right)$ is a Hausdorff uniformizable topological space.

A quasi-metric on a set $X$ is a function $d: X \times X \rightarrow[0,+\infty)$ such that for all $x, y, z \in X:$

(QM1) $d(x, y)=d(y, x)=0 \Leftrightarrow x=y$;

(QM2) $d(x, y) \leq d(x, z)+d(z, y)$.

We shall also consider extended quasi-metrics. These satisfy the preceding conditions (QM1) and (QM2) above, except that we allow $d(x, y)=+\infty$.

By a quasi-metric space we mean a pair $(X, d)$ such that $X$ is a set and $d$ is a quasi-metric or an extended quasi-metric on $X$.

Given an extended quasi-metric $d$ on a set $X$, the function $d^{-1}$ defined on $X \times X$ by $d^{-1}(x, y)=d(y, x)$, is also an extended quasi-metric on $X$, called the conjugate 
of $d$, and the function $d^{s}$ defined on $X \times X$ by $d^{s}(x, y)=\max \left\{d(x, y), d^{-1}(x, y)\right\}$ is an extended metric on $X$. Of course, $d^{-1}$ and $d^{s}$ are a quasi-metric and a metric, respectively, whenever $d$ is a quasi-metric on $X$. Obviously, each (extended) metric $d$ is an (extended) quasi-metric where $d=d^{-1}$.

The following is an easy but useful example of a quasi-metric space.

Example 1.1. Let $d$ be the function defined on $\mathbb{R} \times \mathbb{R}$ by $d(x, y)=\max \{x-y, 0\}$. Then $(\mathbb{R}, d)$ is a quasi-metric space and $d^{s}$ is the Euclidean metric on $\mathbb{R}$.

Each extended quasi-metric $d$ on $X$ induces a $T_{0}$ topology $\tau_{d}$ on $X$ which has as a base the family of open balls $\left\{B_{d}(x, \varepsilon): x \in X, \varepsilon>0\right\}$, where $B_{d}(x, \varepsilon)=\{y \in X$ : $d(x, y)<\varepsilon\}$ for all $x \in X$ and $\varepsilon>0$.

Furthermore, it generates a quasi-uniformity $\mathcal{U}_{d}$ on $X$ which has as a base the countable family $\left\{U_{n}^{d}: n \in \mathbb{N}\right\}$, where $U_{n}^{d}=\left\{(x, y) \in X \times X: d(x, y)<2^{-n}\right\}$ for all $n \in \mathbb{N}$.

A topological space $(X, \tau)$ is said to be quasi-metrizable if there is an extended quasimetric $d$ on $X$ such that $\tau=\tau_{d}$. Similarly, a bispace $\left(X, \tau_{1}, \tau_{2}\right)$ is called quasi-metrizable if there is an extended quasi-metric $d$ on $X$ such that $\tau_{d}=\tau_{1}$ and $\tau_{d^{-1}}=\tau_{2}$.

We conclude this section by recalling several notions, facts and examples on fuzzy (quasi-)metric spaces which we will need in the rest of the paper. Our basic references for fuzzy quasi-metric spaces are [19] and [8].

According to [27], a binary operation $*:[0,1] \times[0,1] \rightarrow[0,1]$ is a continuous t-norm if $*$ satisfies the following conditions: (i) $*$ is associative and commutative; (ii) $*$ is continuous; (iii) $a * 1=a$ for every $a \in[0,1]$; (iv) $a_{1} * b_{1} \leq a_{2} * b_{2}$ whenever $a_{1} \leq a_{2}$ and $b_{1} \leq b_{2}$, with $a_{1}, a_{2}, b_{1}, b_{2} \in[0,1]$.

Three distinguished examples of continuous t-norm are $\wedge$, Prod and $*_{L}$ (the Eukasiewicz t-norm) which are defined as $a \wedge b=\min \{a, b\}, a \operatorname{Prod} b=a b$, and $a *_{L} b=\max \{a+$ $b-1,0\}$ for all $a, b \in[0,1]$, respectively. Recall that $* \leq \wedge$ for every continuous t-norm $*$, and that continuous t-norm greater than or equal to $*_{L}$ is a copula [22].

By a fuzzy quasi-metric (KM-fuzzy quasi-metric in $[8,19]$ ) on a set $X$ we mean a pair $(M, *)$ such that $*$ is a continuous t-norm and $M$ is a fuzzy set in $X \times X \times[0,+\infty)$ such that for all $x, y, z \in X$ :

(FQM1) $M(x, y, 0)=0$;

(FQM2) $M(x, y, t)=M(y, x, t)=1$ for all $t>0 \Leftrightarrow x=y$;

(FQM3) $M(x, z, t+s) \geq M(x, y, t) * M(y, z, s)$ for all $t, s \geq 0$;

$(\mathrm{FQM} 4) M(x, y, \cdot):[0,+\infty) \rightarrow[0,1]$ is left continuous.

If in addition, $M$ satisfies

(FQM5) $M(x, y, t)=M(y, x, t)$ for all $t>0$,

then $(M, *)$ is said to be a fuzzy metric (in the sense of Kramosil and Michalek [23]).

Of course, every fuzzy metric is a fuzzy quasi-metric.

A fuzzy (quasi-)metric space is a triple $(X, M, *)$ such that $X$ is a set and $(M, *)$ is a fuzzy (quasi-)metric on $X$.

It is well known that if $(X, M, *)$ is a fuzzy quasi-metric space, then, for each $x, y \in X$ the function $M(x, y, \cdot)$ is nondecreasing. 
Each fuzzy quasi-metric $(M, *)$ on $X$ induces a $T_{0}$ topology $\tau_{M}$ on $X$ which has as a base the family of open balls $\left\{B_{M}(x, \varepsilon, t): x \in X, \varepsilon \in(0,1), t>0\right\}$, where $B_{M}(x, \varepsilon, t)=\{y \in X: M(x, y, t)>1-\varepsilon\}$ for all $x \in X, \varepsilon \in(0,1)$ and $t>0$.

It is also well known (see e.g. $[17,19]$ ) that every fuzzy (quasi-)metric space is (quasi-)metrizable. More exactly, if $(X, M, *)$ is a fuzzy (quasi-)metric space, then the countable family $\left\{U_{n}: n \in \mathbb{N}\right\}$ is a base for a (quasi-)uniformity $\mathcal{U}_{M}$ on $X$ such that $\tau_{\mathcal{U}_{M}}=\tau_{M}$, where $U_{n}=\{(x, y) \in X \times X: M(x, y, 1 / n)>1-1 / n\}$ for all $n \in \mathbb{N}$.

Example 1.2 (compare [16, Example 2.9], [17, Example 2.16]). Let $d$ be an extended (quasi-)metric on a set $X$ and let $M_{d}$ be the function defined on $X \times X \times[0,+\infty)$ by $M_{d}(x, y, 0)=0$ and

$$
M_{d}(x, y, t)=\frac{t}{t+d(x, y)},
$$

for all $t>0$. (We adopt the convention that $\frac{1}{+\infty}=0$.) Then, for any continuous t-norm $*,\left(M_{d}, *\right)$ is a fuzzy (quasi-)metric on $X$ called the standard fuzzy (quasi-)metric of $(X, d)$, and $\left(X, M_{d}, *\right)$ is called the standard fuzzy (quasi-)metric space of $(X, d)$. Furthermore, it is easy to check that the topologies $\tau_{d}$ and $\tau_{M_{d}}$ coincide on $X$.

Example 1.3 (see e.g. [7, Example 1]). Let $d$ be an extended (quasi-)metric on a set $X$ and let $M_{01}^{d}$ be the function defined on $X \times X \times[0,+\infty)$ by $M_{01}^{d}(x, y, t)=0$ if $d(x, y) \geq t$, and $M_{01}^{d}(x, y, t)=1$ if $d(x, y)<t$. Then, for any continuous t-norm $*,\left(M_{01}^{d}, *\right)$ is a fuzzy (quasi-)metric on $X$. Furthermore, it is easy to check that the topologies $\tau_{d}$ and $\tau_{M_{01}^{d}}$ coincide on $X$.

In the particular case of the metric space $(\mathbb{R}, e)$, where $e$ denotes the Euclidean metric on $\mathbb{R}$, we will simply write $M_{01}$ instead of $M_{01}^{e}$.

Remark 1.1. Let us recall that a sequence $\left(x_{n}\right)_{n \in \mathbb{N}}$ in a fuzzy metric space $(X, M, *)$ is a Cauchy sequence provided that for each $t>0$ and each $\varepsilon \in(0,1)$ there exists $n_{0} \in \mathbb{N}$ such that $M\left(x_{n}, x_{m}, t\right)>1-\varepsilon$ whenever $n, m \geq n_{0}$. A fuzzy metric space $(X, M, *)$ is said to be complete if every Cauchy sequence converges with respect to $\tau_{M}$. It is well known, and easy to check, that if $(X, d)$ is a complete metric space, then the fuzzy metric spaces $\left(X, M_{d}, *\right)$ and $\left(X, M_{01}^{d}, *\right)$, of Examples 1.2 and 1.3, are complete.

\section{The Wijsman topology of a fuzzy metric space}

Following Beer [1, p. 34 and 114], the lower Wijsman topology of a metric space $(X, d)$, denoted by $\tau_{W_{d}}^{-}$, is the weakest topology on $\mathcal{C}_{0}(X)$ for which the functional $d(x, \cdot): \mathcal{C}_{0}(X) \rightarrow[0,+\infty)$ is upper semicontinuous for all $x \in X$. Similarly, the upper Wijsman topology of $(X, d)$, denoted by $\tau_{W_{d}}^{+}$, is the weakest topology on $\mathcal{C}_{0}(X)$ for which the functional $d(x, \cdot)$ is lower semicontinuous for all $x \in X$, and the Wijsman topology of $(X, d)$, denoted by $\tau_{W_{d}}$, is the weakest topology on $\mathcal{C}_{0}(X)$ for which the functional $d(x, \cdot)$ is continuous for all $x \in X$.

Remark 2.1. The topologies $\tau_{W_{d}}^{-}, \tau_{W_{d}}^{+}$and $\tau_{W_{d}}$ always exist because the functional $d(x, \cdot)$ is upper and lower semicontinuous, and hence continuous, for the discrete topology on $\mathcal{C}_{0}(X)$. Moreover, one has $\tau_{W_{d}}=\tau_{W_{d}}^{-} \vee \tau_{W_{d}}^{+}$. 
Definition 2.1. Let $(X, M, *)$ be a fuzzy metric space. The weakest topology on $\mathcal{C}_{0}(X)$ such that for each $x \in X$ and each $t>0$ the functional $M(x, \cdot, t): \mathcal{C}_{0}(X) \rightarrow[0,1]$, given by

$$
M(x, A, t)=\sup _{a \in A} M(x, a, t)
$$

for all $A \in \mathcal{C}_{0}(X)$, is lower semicontinuous will be called the lower Wijsman topology of $(X, M, *)$ and it will be denoted by $\tau_{W_{M}}^{-}$.

Similarly, the weakest topology on $\mathcal{C}_{0}(X)$ such that for each $x \in X$ and each $t>0$ the functional $M(x, \cdot, t)$ is upper semicontinuous will be called the upper Wijsman topology of $(X, M, *)$ and it will be denoted by $\tau_{W_{M}}^{+}$, and the weakest topology on $\mathcal{C}_{0}(X)$ such that for each $x \in X$ and each $t>0$ the functional $M(x, \cdot, t)$ is continuous will be called the Wijsman topology of $(X, M, *)$ and it will be denoted by $\tau_{W_{M}}$.

Remark 2.2. Obviously (compare Remark 2.1) the topologies $\tau_{W_{M}}^{-}, \tau_{W_{M}}^{+}$and $\tau_{W_{M}}$ always exist and one has $\tau_{W_{M}}=\tau_{W_{M}}^{-} \vee \tau_{W_{M}}^{+}$.

Remark 2.3. It is clear that a subbase for $\tau_{W_{M}}^{-}$consists of all sets of the form $\left\{A \in \mathcal{C}_{0}(X): M(x, A, t)>\delta\right\}$, where $x \in X, t>0$ and $0<\delta<1$, while a subbase for $\tau_{W_{M}}^{+}$consists of all sets of the form $\left\{A \in \mathcal{C}_{0}(X): M(x, A, t)<\delta\right\}$, where $x \in X$, $t>0$ and $0<\delta<1$.

Remark 2.4. It follows from Remarks 2.2 and 2.3 that a subbase for $\tau_{W_{M}}$ consists of all sets of the form $\left\{A \in \mathcal{C}_{0}(X): M(x, A, t)>\delta\right\}$ and $\left\{A \in \mathcal{C}_{0}(X): M(x, A, t)<\delta\right\}$, where $x \in X, t>0$ and $0<\delta<1$.

Example 2.1. Let $(X, d)$ be a metric space and let $\left(M_{d}, *\right)$ the fuzzy metric constructed in Example 1.2. Since

$$
M_{d}(x, A, t)=\frac{t}{t+d(x, A)},
$$

for all $x \in X, A \in \mathcal{C}_{0}(X)$ and $t>0$, it immediately follows that the functional $M_{d}(x, \cdot, t)$ is lower semicontinuous (resp. upper semicontinuous) for a topology $\tau$ on $\mathcal{C}_{0}(X)$ if and only if the functional $d(x, \cdot)$ is upper semicontinuous (resp. lower semicontinuous) for $\tau$. Therefore $\tau_{W_{d}}^{-}=\tau_{W_{M_{d}}}^{-}$and $\tau_{W_{d}}^{+}=\tau_{W_{M_{d}}}^{+}$, and hence $\tau_{W_{d}}=\tau_{W_{M_{d}}}$ on $\mathcal{C}_{0}(X)$.

Let us recall (see e.g. [1, p. 6-7]) that the Hausdorff distance on $\mathcal{C}_{0}(X)$ of a metric space $(X, d)$ is the extended metric $H_{d}$ on $\mathcal{C}_{0}(X)$, defined as $H_{d}=\max \left\{H_{d}^{-}, H_{d}^{+}\right\}$, where $H_{d}^{-}$and $H_{d}^{+}$are defined by

$$
H_{d}^{-}(A, B)=\sup _{a \in A} d(a, B), \quad \text { and } \quad H_{d}^{+}(A, B)=\sup _{b \in B} d(A, b),
$$

for all $A, B \in \mathcal{C}_{0}(X)$.

In fact, $H_{d}^{-}$and $H_{d}^{+}$are extended quasi-metrics on $\mathcal{C}_{0}(X)$ (compare $\left.[4,24,25]\right)$. The restriction of $H_{d}^{-}$and $H_{d}^{+}$to the set $\mathcal{C B}_{0}(X)$ of all nonempty closed and bounded, are quasi-metrics and thus $H_{d}$ is a metric on $\mathcal{C B}_{0}(X)$, called the Hausdorff metric of $(X, d)$.

An important and well-known result states that if $(X, d)$ is a metric space, then, for each $x \in X$, the functional $d(x, \cdot)$ is upper semicontinuous on $\left(\mathcal{C}_{0}(X), H_{d}^{-}\right)$and lower semicontinuous on $\left(\mathcal{C}_{0}(X), H_{d}^{+}\right)$, and hence it is continuous on $\left(\mathcal{C}_{0}(X), H_{d}\right)$. Therefore, 
one has $\tau_{W_{d}}^{-} \subseteq \tau_{H_{d}^{-}}, \tau_{W_{d}}^{+} \subseteq \tau_{H_{d}^{+}}$and $\tau_{W_{d}} \subseteq \tau_{H_{d}}$ on $\mathcal{C}_{0}(X)$. Now we will discuss the corresponding situation in the realm of fuzzy metric spaces and we shall show that it has some interesting differences with respect to the classical metric case.

To this end, we first proceed to recall the construction of the Hausdorff fuzzy metric of a fuzzy metric space $(X, M, *)$ (see e.g. [21, p. 98]), which is done by adapting to the fuzzy setting the definition of the Hausdorff probabilistic metric of a probabilistic metric space $[13,27,28,29]$.

For each $A, B \in \mathcal{C}_{0}(X)$ let

$$
\begin{gathered}
H_{M}^{-}(A, B, 0)=H_{M}^{+}(A, B, 0)=0, \\
H_{M}^{-}(A, B, t)=\sup _{0<s<t} \inf _{a \in A} M(a, B, s), \quad H_{M}^{+}(A, B, t)=\sup _{0<s<t} \inf _{b \in B} M(A, b, s),
\end{gathered}
$$

for all $t>0$, and

$$
H_{M}(A, B, t)=\min \left\{H_{M}^{-}(A, B, t), H_{M}^{+}(A, B, t)\right\}
$$

for all $t \geq 0$.

Then $\left(H_{M}, *\right)$ is a fuzzy metric on $\mathcal{C}_{0}(X)$, called the Hausdorff fuzzy metric of $(X, M, *)$. In fact $\left(H_{M}^{-}, *\right)$ and $\left(H_{M}^{+}, *\right)$ are fuzzy quasi-metrics on $\mathcal{C}_{0}(X)[26]$.

Our first result in this section provides a fuzzy extension of the fact that for a metric space $(X, d)$, the functional $d(x, \cdot)$ is upper semicontinuous on $\left(\mathcal{C}_{0}(X), H_{d}^{-}\right)$.

Proposition 2.1. Let $(X, M, *)$ be a fuzzy metric space. Then, for each $x \in X$ and each $t>0$ the functional $M(x, \cdot, t)$ is lower semicontinuous on the fuzzy quasi-metric space $\left(\mathcal{C}_{0}(X), H_{M}^{-}\right)$.

Proof. Suppose that there exist $x \in X$ and $t>0$ for which $M\left(x,{ }_{-}, t\right)$ is not lower semicontinuous on $\left(\mathcal{C}_{0}(X), H_{M}^{-}\right)$. Then, there exist $A \in \mathcal{C}_{0}(X)$ and a sequence $\left(A_{n}\right)_{n \in \mathbb{N}}$ in $\mathcal{C}_{0}(X)$ such that for each $n \in \mathbb{N}$,

$$
\begin{aligned}
& H_{M}^{-}\left(A, A_{n}, 1 / n\right)>1-1 / n, \text { but } \\
& M(x, A, t)-M\left(x, A_{n}, t\right)>\varepsilon \text { for some } \varepsilon \in(0,1) .
\end{aligned}
$$

Since $M(x, A, t)>\varepsilon$ and $M(x, A, \cdot)$ is left continuous, there is $n_{0} \in \mathbb{N}$ such that $M(x, A, t-1 / n)>\varepsilon>1 / n$ for all $n \geq n_{0}$.

Now for each $n \geq n_{0}$ take $y_{n} \in A$ such that

$$
M\left(x, y_{n}, t-1 / n\right)+1 / n>M(x, A, t-1 / n) .
$$

From our assumption that $H_{M}^{-}\left(A, A_{n}, 1 / n\right)>1-1 / n$, it follows that $M\left(y_{n}, A_{n}, 1 / n\right)>$ $1-1 / n$ for all $n \geq n_{0}$, so

$$
\begin{aligned}
M\left(x, A_{n}, t\right) & \geq M\left(x, y_{n}, t-\frac{1}{n}\right) * M\left(y_{n}, A_{n}, \frac{1}{n}\right) \\
& \geq\left(M\left(x, A, t-\frac{1}{n}\right)-\frac{1}{n}\right) *\left(1-\frac{1}{n}\right),
\end{aligned}
$$

for all $n \geq n_{0}$. 
Since $0<M\left(x, A_{n}, t\right)<1$ for all $n \in \mathbb{N}$, there exists a subsequence $\left(A_{n_{k}}\right)_{k \in \mathbb{N}}$ of $\left(A_{n}\right)_{n \in \mathbb{N}}$ for which there exists $\lim _{k} M\left(x, A_{n_{k}}, t\right)$. Then

$$
\lim _{k} M\left(x, A_{n_{k}}, t\right) \geq \lim _{k}\left(M\left(x, A, t-\frac{1}{n_{k}}\right)-\frac{1}{n_{k}}\right) * \lim _{k}\left(1-\frac{1}{n_{k}}\right)=M(x, A, t),
$$

which contradicts our assumption that $M(x, A, t)>M\left(x, A_{n}, t\right)+\varepsilon$ for all $n \in \mathbb{N}$.

As we recalled above, given a metric space $(X, d)$, the functional $d(x, \cdot)$ is lower semicontinuous on $\left(\mathcal{C}_{0}(X), H_{d}^{+}\right)$. The following (compare with Proposition 2.1 above) is an example of a fuzzy metric space $(X, M, *)$ for which there exist $x \in X$ and $t>0$ such that the functional $M(x, \cdot, t)$ is not upper semicontinuous on $\left(\mathcal{C}_{0}(X), H_{M}^{+}\right)$.

Example 2.2. Given the metric space $(\mathbb{R}, e)$, let $\left(M_{01}, \wedge\right)$ be the fuzzy metric on $\mathbb{R}$ associated to $(\mathbb{R}, e)$, as constructed in Example 1.3. Take $x=1, t=1, A=\{0\}$ and $A_{n}=\{1 / n\}$ for all $n \in \mathbb{N}$. Since the sequence $(1 / n)_{n \in \mathbb{N}}$ converges to 0 with respect to the Euclidean topology, i.e., with respect to the topology induced by $\left(M_{01}, \wedge\right)$, then the sequence $\left(A_{n}\right)_{n \in \mathbb{N}}$ converges to $A$ in $\left(\mathcal{C}_{0}(\mathbb{R}), H_{M_{01}}\right)$ and hence in $\left(\mathcal{C}_{0}(\mathbb{R}), H_{M_{01}}^{+}\right)$. Nevertheless, we have $M_{01}(x, A, t)=0$ and $M_{01}\left(x, A_{n}, t\right)=1$ for all $n \in \mathbb{N}$, so $M_{01}(1, \cdot, 1)$ is not upper semicontinuous on $\left(\mathcal{C}_{0}(\mathbb{R}), H_{M_{01}}^{+}\right)$.

From Proposition 2.1 it follows that for any fuzzy metric space $(X, M, *)$ one has $\tau_{W_{M}}^{-} \subseteq \tau_{H_{M}^{-}}$on $\mathcal{C}_{0}(X)$. Example 2.2 shows that, nevertheless, the inclusion $\tau_{W_{M}}^{+} \subseteq \tau_{H_{M}^{+}}$ does not hold in general. However we can show the following result.

Proposition 2.2. Let $(X, M, *)$ be a fuzzy metric space such that for each $x \in X$ and each $A \in \mathcal{C}_{0}(X)$ the function $M(x, A, \cdot):(0,+\infty) \rightarrow[0,1]$ is continuous. Then, for each $x \in X$ and each $t>0$ the functional $M(x, \cdot, t)$ is upper semicontinuous on the fuzzy quasi-metric space $\left(\mathcal{C}_{0}(X), H_{M}^{+}\right)$.

Proof. Suppose that there exist $x \in X$ and $t>0$ for which $M(x, \cdot, t)$ is not upper semicontinuous on $\left(\mathcal{C}_{0}(X), H_{M}^{+}\right)$. Then, there exist $A \in \mathcal{C}_{0}(X)$ and a sequence $\left(A_{n}\right)_{n \in \mathbb{N}}$ in $\mathcal{C}_{0}(X)$ such that for each $n \in \mathbb{N}$,

$$
\begin{aligned}
& H_{M}^{+}\left(A, A_{n}, 1 / n\right)>1-1 / n, \text { but } \\
& M\left(x, A_{n}, t\right)-M(x, A, t)>\varepsilon \text { for some } \varepsilon \in(0,1) .
\end{aligned}
$$

For each $n \in \mathbb{N}$ take $a_{n} \in A_{n}$ such that

$$
M\left(x, a_{n}, t\right)+1 / n>M\left(x, A_{n}, t\right) .
$$

From our assumption that $H_{M}^{+}\left(A, A_{n}, 1 / n\right)>1-1 / n$, it follows that $M\left(A, a_{n}, 1 / n\right)>$ $1-1 / n$ for all $n \in \mathbb{N}$.

Now choose $n_{0} \in \mathbb{N}$ with $\varepsilon>1 / n_{0}$. Then for each $n \geq n_{0}$ we have

$$
\begin{aligned}
M\left(x, A, t+\frac{1}{n}\right) & \geq M\left(x, a_{n}, t\right) * M\left(a_{n}, A, \frac{1}{n}\right) \\
& \geq\left(M\left(x, A_{n}, t\right)-\frac{1}{n}\right) *\left(1-\frac{1}{n}\right) .
\end{aligned}
$$


Since $M\left(x, A_{n}, t\right)-1 / n \in(0,1)$ for all $n \geq n_{0}$ there exist a subsequence $\left(A_{n_{k}}\right)_{k \in \mathbb{N}}$ of $\left(A_{n}\right)_{n \in \mathbb{N}}$ and a real number $r \in[0,1]$ such that

$$
\lim _{k \rightarrow \infty} M\left(x, A_{n_{k}}, t\right)=r .
$$

Hence, from our hypothesis that $M(x, A, \cdot)$ is continuous on $(0,+\infty)$, and taking into account that $*$ is continuous on $[0,1] \times[0,1]$, we deduce that

$$
M(x, A, t) \geq r
$$

Therefore $M\left(x, A_{n}, t\right)>\varepsilon+r$ for all $n \in \mathbb{N}$, which contradicts that $\lim _{k \rightarrow \infty} M\left(x, A_{n_{k}}, t\right)=$ $r$.

From Remak 2.2, and Propositions 2.1 and 2.2 we deduce the following.

Corollary 2.1. Let $(X, M, *)$ be a fuzzy metric space such that for each $x \in X$ and each $A \in \mathcal{C}_{0}(X)$ the function $M(x, A, \cdot):(0,+\infty) \rightarrow[0,1]$ is continuous. Then, for each $x \in X$ and each $t>0$ the functional $M(x, \cdot, t)$ is continuous on the fuzzy metric space $\left(\mathcal{C}_{0}(X), H_{M}\right)$.

Note that the function $M(x, A, \cdot):(0,+\infty) \rightarrow[0,1]$ is left continuous (equivalently, lower semicontinuous) for each $x \in X$ and each $A \in \mathcal{C}_{0}(X)$. Consequently, in Proposition 2.2 and Corollary 2.1 it would be enough to ask that the function $M(x, A, \cdot)$ is right continuous (equivalently, upper semicontinuous) for each $x \in X$ and each $A \in \mathcal{C}_{0}(X)$..

In the sequel a fuzzy metric space $(X, M, *)$ such that for each $x \in X$ and each $A \in \mathcal{C}_{0}(X)$ the function $M(x, A, \cdot):(0,+\infty) \rightarrow[0,1]$ is upper semicontinuous (equivalently, continuous), will be called a $C$-usc fuzzy metric space. In such a case, we will say that $(M, *)$ is a $C$-usc fuzzy metric on $X$.

Remark 2.5. Although it is clear that the fuzzy metric space of Example 2.2 is not $C$-usc, there exist several interesting examples of $C$-usc fuzzy metric spaces. In fact, the standard fuzzy metric space (Example 2.1), and any stationary fuzzy metric space are instances of $C$-usc fuzzy metric spaces (recall that a fuzzy metric $(M, *)$ on a set $X$ is stationary [18] provided that for each $x, y \in X$, the function $M\left(x, y,{ }_{-}\right):[0,+\infty) \rightarrow[0,1]$ is constant). For another example, let $(X, d)$ be a metric space; then, it is easy to check that $(X, M, *)$ is a $C$-usc fuzzy metric space, where $*$ is any continuous tnorm and $M$ is defined on $X \times X \times[0,+\infty)$ by $M(x, y, t)=0$ if $d(x, y) \geq t$, and $M(x, y, t)=(t-d(x, y)) /(t+d(x, y))$ if $d(x, y)<t$.

The following two results provide fundamental properties of the Wijsman topology of a metric space (see e.g. [1, Lemma 2.1.4 and Theorem 2.1.5]).

For a metric space $(X, d)$ the following hold:

(A) The Wijsman topology $\tau_{W_{d}}$ on $\mathcal{C}_{0}(X)$ is admissible, Hausdorff and uniformizable.

(B) $\tau_{W_{d}}$ on $\mathcal{C}_{0}(X)$ is second countable if and only if $(X, d)$ is separable.

Next we discuss the corresponding properties in the realm of fuzzy metric spaces. We shall show that, extending parts of result (A) above, the Wijsman topology $\tau_{W_{M}}$ on $\mathcal{C}_{0}(X)$ of a fuzzy metric space $(X, M, *)$ is Hausdorff and uniformizable (actually, 
we shall show a more general result involving quasi-uniformizability of the lower and upper Wijsman topologies). On the other hand, we shall show that contrarily to the metric case, the space of Example 2.2 provides an instance of a separable fuzzy metric space for which its Wijsman topology is not admissible and does not admit a countable base. However, we are able to show that separability of a fuzzy metric space $(X, M, *)$ characterizes both second countability and quasi-metrizability of the lower Wijsman topology on $\mathcal{C}_{0}(X)$. We will conclude this section by showing that if $(X, M, *)$ is a $C$-usc fuzzy metric space, then the following conditions are equivalent: (i) the lower and upper Wijsman topologies on $\mathcal{C}_{0}(X)$ are second countable; (ii) the Wijsman topology on $\mathcal{C}_{0}(X)$ is second countable; (iii) $(X, M, *)$ is separable. The classical metric result (B) will be deduced as a consequence of that characterization.

Of course, a fuzzy metric space $(X, M, *)$ is said to be separable if the metrizable topological space $\left(X, \tau_{M}\right)$ is separable (equivalently, if $\tau_{M}$ has a countable base).

Theorem 2.1. Let $(X, M, *)$ be a fuzzy metric space. Then $\left(\mathcal{C}_{0}(X), \tau_{W_{M}}^{-}, \tau_{W_{M}}^{+}\right)$is a quasi-uniformizable $T_{0}$-bispace.

Proof. It straightforward to see that $\tau_{W_{M}}^{-}$is a $T_{0}$ topology on $\mathcal{C}_{0}(X)$.

Now, for each $x \in X, \varepsilon \in(0,1)$ and $t>0$ define

$$
U_{x, \varepsilon, t}=\left\{(A, B) \in \mathcal{C}_{0}(X) \times \mathcal{C}_{0}(X): M(x, A, t)-M(x, B, t)<\varepsilon\right\} .
$$

Then, it is routine to check that the collection

$$
\left\{U_{x, \varepsilon, t}: x \in X, \varepsilon \in(0,1), t>0\right\},
$$

generates a quasi-uniformity $\mathcal{U}_{W_{M}}$ on $\mathcal{C}_{0}(X)$. Now let $\left(A_{\lambda}\right)_{\lambda \in \Lambda}$ be a net in $\mathcal{C}_{0}(X)$. By Definition 2.1, $\left(A_{\lambda}\right)_{\lambda \in \Lambda} \tau_{W_{M}}^{-}$-converges to an $A \in \mathcal{C}_{0}(X)$ if and only if for each $x \in X$, $\varepsilon \in(0,1)$ and $t>0$, we have $A_{\lambda} \in U_{x, \varepsilon, t}(A)$ eventually. We deduce that $\tau_{\mathcal{U}_{W_{M}}}=\tau_{W_{M}}^{-}$. A similar argument shows that $\tau_{\mathcal{U}_{W_{M}}^{-1}}=\tau_{W_{M}}^{+}$.

Corollary 2.2. Let $(X, M, *)$ be a fuzzy metric space. Then $\left(\mathcal{C}_{0}(X), \tau_{W_{M}}\right)$ is a Hausdorff uniformizable space.

As we recalled above, an important fact in the realm of metric spaces is that the Wijsman topology on $\mathcal{C}_{0}(X)$ of a metric space $(X, d)$ is admissible, i.e., the relative topology, denoted by $\tau_{W_{d} \mid X}$, that $X$ inherits from $\tau_{W_{d}}$, under the identification $x \leftrightarrow\{x\}$, agrees with the topology $\tau_{d}$ induced by $d$ (see e.g. [1, Lemma 2.1.4]).

We shall show that this fact does not hold for fuzzy metric spaces, in general. More exactly, we shall show that for the fuzzy metric $\left(M_{01}, \wedge\right)$ of Example 2.2, the relative topology $\tau_{W_{M_{01}} \mid \mathbb{R}}$, that $\mathbb{R}$ inherits from the Wijsman topology $\tau_{W_{M_{01}}}$ is the discrete topology on $\mathbb{R}$. Therefore, $\tau_{W_{M_{01}}}$ on $\mathcal{C}_{0}(X)$ is not second countable, so result (B) cannot be generalized to fuzzy metric spaces without some additional condition.

The following auxiliary result, whose easy proof we omit, will be useful.

Lemma 2.1. Let $(X, M, *)$ be a fuzzy metric space. Then $\tau_{W_{M} \mid X}^{-}=\tau_{M}$.

Example 2.3. Consider the fuzzy metric $\left(M_{01}, \wedge\right)$ associated to the (Euclidean) metric space $(\mathbb{R}, e)$. Then, for each $x, y \in \mathbb{R}$ and $t \geq 0, M_{01}(x, y, t)=1$ if $|x-y|<t$, and 
$M_{01}(x, y, t)=0$ if $|x-y| \geq t$. Since $(\mathbb{R}, e)$ is separable, then $\left(\mathbb{R}, M_{01}, \wedge\right)$ is separable. In particular $\tau_{e}=\tau_{M_{01}}$, so by Lemma $2.1, \tau_{W_{M_{01}}}^{-} \mid \mathbb{R}$ is the Euclidean topology on $\mathbb{R}$. Now take any $x \in \mathbb{R}$. Then (see the construction in the proof of Theorem 2.1) for each $\varepsilon \in(0,1)$ we obtain

$$
\begin{aligned}
\left(U_{x-1, \varepsilon, 1}\right)^{-1}(x) & =\left\{y \in \mathbb{R}: M_{01}(x-1, y, 1)-M_{01}(x-1, x, 1)<\varepsilon\right\} \\
& =\left\{y \in \mathbb{R}: M_{01}(x-1, y, 1)<\varepsilon\right\} \\
& =\left\{y \in \mathbb{R}: M_{01}(x-1, y, 1)=0\right\} \\
& =\{y \in \mathbb{R}:|x-1-y| \geq 1\}=(-\infty, x-2] \cup[x, \infty) .
\end{aligned}
$$

Similarly

$$
\begin{aligned}
\left(U_{x-1, \varepsilon, 1}\right)^{-1}(x) & =\left\{y \in \mathbb{R}: M_{01}(x+1, y, 1)<\varepsilon\right\} \\
& =\{y \in \mathbb{R}:|x+1-y| \geq 1\}=(-\infty, x] \cup[x+2, \infty),
\end{aligned}
$$

where the entourages $U_{x-1, \varepsilon, 1}$ and $U_{x-1, \varepsilon, 1}$ are restricted to $\mathbb{R} \times \mathbb{R}$.

Since by Theorem 2.1 and its proof, $\left(U_{x-1, \varepsilon, 1}\right)^{-1}(x)$ and $\left(U_{x+1, \varepsilon, 1}\right)^{-1}(x)$ are $\tau_{W_{M_{01}}}^{+} \mathbb{R}^{-}$ neighborhoods of $x$, we deduce that $V(x)=\left(U_{x-1, \varepsilon, 1}\right)^{-1}(x) \cap\left(U_{x+1, \varepsilon, 1}\right)^{-1}(x)$ is also a

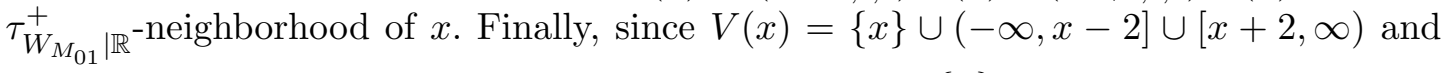
$\tau_{W_{M_{01}} \mid \mathbb{R}}^{-}$is the Euclidean topology on $\mathbb{R}$ we deduce that $\{x\}$ is a $\tau_{W_{M_{01}} \mid \mathbb{R}^{-n e i g h b o r h o o d}}$ of $x$. We conclude that $\tau_{W_{M_{01}} \mid \mathbb{R}}$ is the discrete topology on $\mathbb{R}$.

In the proof of our next result we adapt a technique used for the case of metric spaces (see e.g. [1, Theorem 2.1.5]) to our fuzzy framework. In particular, we will need the following adaptation of a well-known procedure for $\varepsilon$-discrete subsets of a metric space $[1$, p. 36].

Let $\varepsilon \in(0,1)$. A subset $E$ of a fuzzy metric space $(X, M, *)$ is said to be $\varepsilon$-discrete if for each $x, y \in E$, with $x \neq y$, we have $M(x, y, \varepsilon) \leq 1-\varepsilon$. Denote by $\mathcal{E}$ the collection of all $\varepsilon$-discrete subsets of $(X, M, *)$ ordered by set inclusion. Since for any linearly ordered subset $\mathcal{L}$ on $\mathcal{E}$, the $\varepsilon$-discrete set $\cup_{E \in \mathcal{L}} E$ is an upper bound of $\mathcal{L}$, we deduce from Zorn's lemma that $X$ has a maximal $\varepsilon$-discrete subset $E_{\varepsilon}$. Furthermore $X=\cup_{x \in E_{\varepsilon}} B_{M}(x, \varepsilon, \varepsilon)$. Indeed, if there is $y \in X \backslash \cup_{x \in E_{\varepsilon}} B_{M}(x, \varepsilon, \varepsilon)$, then $\{y\} \cup E_{\varepsilon}$ is a $\varepsilon$-discrete subset of $X$, which contradicts the fact that $E_{\varepsilon}$ is maximal.

Theorem 2.2. For a fuzzy metric space $(X, M, *)$ the following conditions are equivalent:

(1) $(X, M, *)$ is separable;

(2) The topology $\tau_{W_{M}}^{-}$on $\mathcal{C}_{0}(X)$ is second countable;

(3) $\left(\mathcal{C}_{0}(X), \tau_{W_{M}}^{-}\right)$is a quasi-metrizable space;

(4) The topology $\tau_{W_{M}}^{-}$on $\mathcal{C}_{0}(X)$ is first countable.

Proof. $(1) \Rightarrow(2)$. Let $D$ be a countable dense subset of $\left(X, \tau_{M}\right)$. We shall show that if we denote by $\mathbb{Q}^{+}$the set of all positive rational numbers, then the sets

$$
\left\{A \in \mathcal{C}_{0}(X): M(x, A, t)>\delta\right\}, \quad\left(x \in D, t \in \mathbb{Q}^{+}, \delta \in(0,1) \cap \mathbb{Q}^{+}\right),
$$

form a countable subbase of the topology $\tau_{W_{M}}^{-}$on $\mathcal{C}_{0}(X)$. 
To this end, let $A \in \mathcal{C}_{0}(X), x \in X, t>0$ and $\delta \in(0,1)$ such that $A \in V$, where

$$
V=\left\{B \in \mathcal{C}_{0}(X): M(x, B, t)>\delta\right\} .
$$

Choose $\delta_{1}, \delta_{2} \in(0, \delta) \cap \mathbb{Q}^{+}$such that $M(x, A, t)>\delta_{2}>\delta_{1}>\delta$. There exists $a_{0} \in A$ such that $M\left(x, a_{0}, t\right)>\delta_{2}$.

By the left continuity of $M\left(x, a_{0}, \cdot\right)$, there exists $s_{0} \in(0, t)$ such that $M\left(x, a_{0}, s\right)>\delta_{2}$ whenever $s \in\left[s_{0}, t\right]$. Choose $\eta \in(0,1)$ and $t_{1} \in \mathbb{Q}^{+}$such that $s_{0}+2 \eta<t_{1}+\eta<t$.

Now take $\varepsilon \in(0,1)$ such that $(1-\varepsilon) * \delta_{2}>\delta_{1}$ and $(1-\varepsilon) * \delta_{1}>\delta$. Then, there exists $z \in D$ such that $M(x, z, \eta)>1-\varepsilon$.

We show that $M\left(z, A, t_{1}\right)>\delta_{1}$. Indeed, we have

$$
M\left(z, A, t_{1}\right) \geq M\left(z, a_{0}, t_{1}\right) \geq M(z, x, \eta) * M\left(x, a_{0}, s_{0}\right) \geq(1-\varepsilon) * \delta_{2}>\delta_{1} .
$$

Finally, let $B \in \mathcal{C}_{0}(X)$ such that $M\left(z, B, t_{1}\right)>\delta_{1}$. Then, there exists $b_{0} \in B$ such that $M\left(z, b_{0}, t_{1}\right)>\delta_{1}$. Hence

$$
M(x, B, t) \geq M\left(x, b_{0}, t\right) \geq M(x, z, \eta) * M\left(z, b_{0}, t_{1}\right) \geq(1-\varepsilon) * \delta_{1}>\delta,
$$

and thus $B \in V$. This concludes the proof.

$(2) \Rightarrow(3)$. This follows from the well-known result (see e.g. [9, Theorems 1.1.25 and 1.2.53]) that every $T_{0}$ second countable topological space is quasi-metrizable.

$(3) \Rightarrow(4)$ is obvious.

$(4) \Rightarrow(1)$. If for each $\varepsilon \in(0,1)$, any $\varepsilon$-discrete subset of $(X, M, *)$ were countable, then for each $n \in \mathbb{N}$ there would exist a countable $1 / n$-discrete subset $E_{n}$ of $X$ such that $X=\cup_{x \in E_{n}} B_{M}(x, 1 / n, 1 / n)$. Then $X=\overline{\cup_{n \in \mathbb{N}} E_{n}}$, so $(X, M, *)$ would be separable. Hence, if $(X, M, *)$ is not separable, there exist an $\varepsilon \in(0,1)$ and an uncountable $\varepsilon$ discrete subset $W$ of $X$. Clearly, a base of $\tau_{W_{M}}^{-}$-neighborhoods of $X \in \mathcal{C}_{0}(X)$ consists of all sets of the form

$$
\mathcal{B}(F, t, \delta)=\left\{A \in \mathcal{C}_{0}(X): M(x, A, t)>\delta \text { for all } x \in F\right\},
$$

where $F$ runs over the finite subsets of $X, t>0$ and $\delta \in(0,1)$.

Let $\left\{\mathcal{B}\left(F_{n}, t_{n}, \delta_{n}\right): n \in \mathbb{N}\right\}$ be a countable family of such sets. Choose $\delta \in(0,1)$ such that $(1-\delta) *(1-\delta)>1-\varepsilon$.

Then, there exists $w_{0} \in W$ such that $M\left(w_{0}, x, \varepsilon / 2\right)<1-\delta$ for all $x \in \cup_{n \in \mathbb{N}} F_{n}$.

(Otherwise, since $\cup_{n \in \mathbb{N}} F_{n}$ is countable and $W$ is uncountable, there are $w_{1}, w_{2} \in W$, $w_{1} \neq w_{2}$, and $x_{0} \in X$ such that $M\left(w_{i}, x_{0}, \varepsilon / 2\right) \geq 1-\delta, i=1,2$. Thus, $M\left(w_{1}, w_{2}, \varepsilon\right)>$ $1-\varepsilon$, which contradicts the fact that $W$ is $\varepsilon$-discrete.)

Finally, consider the $\tau_{W_{M}}^{-}$-neighborhood of $X$,

$$
U_{X}=\left\{A \in \mathcal{C}_{0}(X): M\left(w_{0}, A, \varepsilon / 2\right)>1-\delta\right\} .
$$

Since for each $n \in \mathbb{N}, F_{n} \in \mathcal{B}\left(F_{n}, t_{n}, \delta_{n}\right) \backslash U_{X}$, we deduce that $X$ has no a countable base of $\tau_{W_{M}}^{-}$-neighborhoods, and, hence, the topology $\tau_{W_{M}}^{-}$fails to be first countable. 
Theorem 2.3. For a $C$-usc fuzzy metric space $(X, M, *)$ the following conditions are equivalent:

(1) $(X, M, *)$ is separable;

(2) The topologies $\tau_{W_{M}}^{-}$and $\tau_{W_{M}}^{+}$on $\mathcal{C}_{0}(X)$ are second countable;

(3) $\left(\mathcal{C}_{0}(X), \tau_{W_{M}}^{-}, \tau_{W_{M}}^{+}\right)$is a quasi-metrizable bispace;

(4) The topologies $\tau_{W_{M}}^{-}$and $\tau_{W_{M}}^{+}$on $\mathcal{C}_{0}(X)$ are first countable.

Proof. $(1) \Rightarrow(2)$. Let $D$ be a countable dense subset of $\left(X, \tau_{M}\right)$. Then, the sets

$$
\left\{A \in \mathcal{C}_{0}(X): M(x, A, t)>\delta\right\}, \quad\left(x \in D, t \in \mathbb{Q}^{+}, \delta \in(0,1) \cap \mathbb{Q}^{+}\right),
$$

and

$$
\left\{A \in \mathcal{C}_{0}(X): M(x, A, t)<\delta\right\}, \quad\left(x \in D, t \in \mathbb{Q}^{+}, \delta \in(0,1) \cap \mathbb{Q}^{+}\right),
$$

form countable subbases of the topologies $\tau_{W_{M}}^{-}$and $\tau_{W_{M}}^{+}$on $\mathcal{C}_{0}(X)$, respectively.

Indeed, it was proved in Theorem $2.2,(1) \Rightarrow(2)$, that the sets

$$
\left\{A \in \mathcal{C}_{0}(X): M(x, A, t)>\delta\right\}, \quad\left(x \in D, t \in \mathbb{Q}^{+}, \delta \in(0,1) \cap \mathbb{Q}^{+}\right),
$$

form a countable subbase of $\tau_{W_{M}}^{-}$on $\mathcal{C}_{0}(X)$.

In order to complete the proof of this implication let $A \in \mathcal{C}_{0}(X), x \in X, t>0$ and $\delta \in(0,1)$ such that $A \in V$, where

$$
V=\left\{B \in \mathcal{C}_{0}(X): M(x, B, t)<\delta\right\} .
$$

Choose $\delta_{1} \in(0, \delta) \cap \mathbb{Q}^{+}$such that $M(x, A, t)<\delta_{1}$. Since $(X, M, *)$ is $C$-usc there exist $t_{1} \in \mathbb{Q}^{+}$, with $t_{1}>t$, and $\varepsilon>0$ such that $M\left(x, A, t^{\prime}\right)<\delta_{1}$ whenever $t^{\prime} \in\left[t, t_{1}+\varepsilon\right]$.

Take $\delta_{2} \in(0,1)$ such that $M\left(x, A, t_{1}+\varepsilon\right)<\delta_{1} * \delta_{2}$, and $\delta_{3} \in(0,1)$ such that $\delta_{3} * \delta>\delta_{1}$.

Now take $t^{\prime} \in(t, t+\varepsilon)$ with $t^{\prime}<t_{1}$. Then, there is $z \in D$ such that $M\left(x, z, t^{\prime}-t\right)>$ $\max \left\{\delta_{2}, \delta_{3}\right\}$.

We show that $M\left(z, A, t_{1}\right)<\delta_{1}$. Assume the contrary. Then there is a sequence $\left(a_{n}\right)_{n}$ in $A$ such that

$$
M\left(z, a_{n}, t_{1}\right)+\frac{1}{n}>\delta_{1}
$$

eventually. Therefore

$$
M\left(x, a_{n}, t_{1}+\varepsilon\right) \geq M\left(x, z, t^{\prime}-t\right) * M\left(z, a_{n}, t_{1}\right) \geq \delta_{2} *\left(\delta_{1}-\frac{1}{n}\right),
$$

eventually. Consequently

$$
M\left(x, A, t_{1}+\varepsilon\right) \geq \delta_{2} * \delta_{1},
$$

which contradicts the fact that $M\left(x, A, t_{1}+\varepsilon\right)<\delta_{1} * \delta_{2}$.

It remains to show that $B \in V$ whenever $B \in \mathcal{C}_{0}(X)$ satisfies $M\left(z, B, t_{1}\right)<\delta_{1}$. Indeed, suppose that for such a $B$ one has $M(x, B, t) \geq \delta$. Then there exists a sequence $\left(b_{n}\right)_{n}$ in $B$ such that

$$
M\left(x, b_{n}, t\right)+\frac{1}{n}>\delta
$$

eventually. Therefore

$$
M\left(z, b_{n}, t_{1}\right) \geq M\left(z, x, t^{\prime}-t\right) * M\left(x, b_{n}, t\right) \geq \delta_{3} *\left(\delta-\frac{1}{n}\right),
$$


eventually. Consequently

$$
M\left(z, B, t_{1}\right) \geq \delta_{3} * \delta>\delta_{1},
$$

which contradicts the fact that $M\left(z, B, t_{1}\right)<\delta_{1}$. We conclude that $B \in V$.

$(2) \Rightarrow(3)$. This follows from Theorem 2.1 and the well-known result (see e.g. $[9$, Theorems 1.1.25 and 1.2.53]) that every quasi-uniformizable $T_{0}$-bispace $\left(X, \tau_{1}, \tau_{2}\right)$ such that $\tau_{1}$ and $\tau_{2}$ are second countable topologies, is quasi-metrizable.

$(3) \Rightarrow(4)$ is obvious and $(4) \Rightarrow(1)$ follows from Theorem $2.2,(4) \Rightarrow(1)$.

Corollary 2.3. For a $C$-usc fuzzy metric space $(X, M, *)$ the following conditions are equivalent:

(1) $(X, M, *)$ is separable;

(2) The topology $\tau_{W_{M}}$ on $\mathcal{C}_{0}(X)$ is second countable;

(3) $\left(\mathcal{C}_{0}(X), \tau_{W_{M}}\right)$ is a metrizable space;

(4) The topology $\tau_{W_{M}}$ on $\mathcal{C}_{0}(X)$ is first countable.

From Corollary 2.3, Example 2.1 and Remark 2.5 we deduce the following.

Corollary 2.4 (see e.g. [1, Theorem 2.1.5]). For a metric space $(X, d)$ the following conditions are equivalent:

(1) $(X, d)$ is separable;

(2) The topology $\tau_{W_{d}}$ on $\mathcal{C}_{0}(X)$ is second countable;

(3) $\left(\mathcal{C}_{0}(X), \tau_{W_{d}}\right)$ is a metrizable space;

(4) The topology $\tau_{W_{d}}$ on $\mathcal{C}_{0}(X)$ is first countable.

Remark 2.6. A very nice result due to Beer (see [1, Theorem 2.5.4]) states that if $(X, d)$ is a complete and separable metric space, then $\left(\mathcal{C}_{0}(X), \tau_{W_{d}}\right)$ is separable and admits a compatible complete metric. Example 2.3 shows that this result cannot be generalized to fuzzy metric spaces, in general. Indeed, since $(\mathbb{R}, e)$ is complete and separable, then $\left(\mathbb{R}, M_{01}, \wedge\right)$ is complete and separable but $\left(\mathcal{C}_{0}(X), \tau_{W_{M_{01}}}\right)$ is not separable because, obviously, $\left(\mathbb{R}, \tau_{W_{M_{01}} \mid \mathbb{R}}\right)$ is not separable (recall that every subspace of a separable metrizable space is separable).

Remark 2.7. It seems interesting to point out that Theorem 2.2 (3) and Example 2.3 provide an instance of a quasi-metric $d$ on $\mathbb{R}$ such that $\tau_{d}$ is the Euclidean topology on $\mathbb{R}$ and $\tau_{d^{s}}$ is the discrete topology on $\mathbb{R}$.

\section{Connections with other hypertopologies}

In this section we explore the relationship between the Wijsman topology of a fuzzy metric space $(X, M, *)$ and other topologies on $\mathcal{C}_{0}(X)$ that arise in a natural way in this context.

We first describe convergence in the Wijsman topology in a similar way to the classical metric case (compare [1, Lemma 2.1.2]).

Proposition 3.1. Let $(X, M, *)$ be a fuzzy metric space. Then a net $\left(A_{\lambda}\right)_{\lambda \in \Lambda}$ in $\mathcal{C}_{0}(X)$ is: 
(a) $\tau_{W_{M}}^{-}$-convergent to $A \in \mathcal{C}_{0}(X)$ if and only if whenever $A \cap G \neq \varnothing$ then $A_{\lambda} \cap G \neq \varnothing$ eventually for every nonempty open set $G$;

(b) $\tau_{W_{M}}^{+}$-convergent to $A \in \mathcal{C}_{0}(X)$ if and only if whenever $0<\delta<\varepsilon<1$ and $t>0$ then $B_{M}(x, \varepsilon, t) \cap A=\varnothing$ implies $B_{M}(x, \delta, t) \cap A_{\lambda}=\varnothing$ eventually.

Proof. (a) Suppose that $\left(A_{\lambda}\right)_{\lambda \in \Lambda}$ is $\tau_{W_{M}}^{-}$-convergent to $A$. Let $G$ be a nonempty open set such that there exists $x \in A \cap G$. Let $\varepsilon \in(0,1)$ and $t>0$ such that $B_{M}(x, \varepsilon, t) \subseteq G$. Since $A \in\left\{B \in \mathcal{C}_{0}(X): M(x, B, t)>1-\varepsilon\right\}$, then $A_{\lambda}$ belongs to that set eventually, i.e., $M\left(x, A_{\lambda}, t\right)>1-\varepsilon$ so $B_{M}(x, \varepsilon, t) \cap A_{\lambda} \neq \varnothing$ eventually.

Conversely, suppose that $A \in\left\{B \in \mathcal{C}_{0}(X): M(x, B, t)>\varepsilon\right\}$. Then $A \cap B_{M}(x, 1-$ $\varepsilon, t) \neq \varnothing$ so $A_{\lambda} \cap B_{M}(x, 1-\varepsilon, t) \neq \varnothing$ eventually. Consequently, $A_{\lambda} \in\left\{B \in \mathcal{C}_{0}(X)\right.$ : $M(x, B, t)>\varepsilon\}$ eventually.

(b) Suppose that $\left(A_{\lambda}\right)_{\lambda \in \Lambda}$ is $\tau_{W_{M}}^{+}$-convergent to $A$. Let $0<\delta<\varepsilon<1$ and $t>0$ with $B_{M}(x, \varepsilon, t) \cap A=\varnothing$. Then $M(x, A, t) \leq 1-\varepsilon<1-\delta$. By assumption $M\left(x, A_{\lambda}, t\right)<1-\delta$ eventually, so $B_{M}(x, \delta, t) \cap A_{\lambda}=\varnothing$ eventually.

Conversely, suppose that $A \in\left\{B \in \mathcal{C}_{0}(X): M(x, B, t)<\varepsilon\right\}$. Let $\alpha=M(x, A, t)<\varepsilon$. Since $B_{M}(x, 1-\alpha, t) \cap A=\varnothing$, by hypothesis $B_{M}\left(x, 1-\frac{\alpha+\varepsilon}{2}, t\right) \cap A_{\lambda}=\varnothing$, i.e., $M\left(x, A_{\lambda}, t\right) \leq(\alpha+\varepsilon) / 2<\varepsilon$ eventually.

Remark 3.1. We observe that as an immediate consequence of part (a) of the above result, the lower Wijsman topology of a fuzzy metric space $(X, M, *)$ coincides with the lower Vietoris topology [1] associated with the topology $\tau_{M}$ which has as a base the elements of the form

$$
G^{-}=\left\{A \in \mathcal{C}_{0}(X): A \cap G \neq \varnothing\right\},
$$

where $G$ is a nonempty $\tau_{M}$-open subset of $X$.

Indeed, it is easy to prove that if $G$ is $\tau_{M}$-open and for each $x \in G$ we pick up $\varepsilon_{x} \in(0,1)$ and $t_{x}>0$ such that $B_{M}\left(x, \varepsilon_{x}, t_{x}\right) \subseteq G$, then

$$
G^{-}=\cup_{x \in G}\left\{A \in \mathcal{C}_{0}(X): M\left(x, A, t_{x}\right)>1-\varepsilon_{x}\right\} .
$$

Moreover, it is obvious that

$$
\left\{A \in \mathcal{C}_{0}(X): M(x, A, t)>1-\varepsilon\right\}=\left(B_{M}(x, \varepsilon, t)\right)^{-} .
$$

From this fact and Theorem 2.2 it follows that a fuzzy metric space $(X, M, *)$ is separable if and only if the lower Vietoris topology associated to $\tau_{M}$ is second countable on $\mathcal{C}_{0}(X)$.

Next we recall two definitions of classical hypertopologies ( see e.g. [1]).

Let $(X, \tau)$ be a topological space. The upper Vietoris topology $\tau_{V}^{+}$on $\mathcal{C}_{0}(X)$ is the topology generated by all sets of the form

$$
G^{+}=\left\{B \in \mathcal{C}_{0}(X): B \subseteq G\right\},
$$

where $G$ is a nonempty open subset of $X$.

Let $(X, \mathcal{U})$ be a uniform space. The upper proximal topology $\sigma_{\mathcal{U}}^{++}$on $\mathcal{C}_{0}(X)$ is the topology generated by all sets of the form

$$
G^{++}=\left\{B \in \mathcal{C}_{0}(X): U(B) \subseteq G \text { for some } U \in \mathcal{U}\right\},
$$


where $G$ is a nonempty open subset of $X$.

It is clear that for every uniform space $(X, \mathcal{U}), \sigma_{\mathcal{U}}^{++} \subseteq \tau_{V}^{+}$, where $\tau_{V}^{+}$is the upper Vietoris topology of $\left(X, \tau_{\mathcal{U}}\right)$.

If $(X, d)$ is a metric space we shall denote by $\sigma_{d}^{++}$the upper proximal topology of the uniformity generated by $d$.

Observe that the definition of the upper Vietoris topology is purely topological so it can be considered directly in a fuzzy metric space. Nevertheless, the definition of the upper proximal topology makes use of a uniformity so a suitable definition is needed in this framework.

Definition 3.1. Let $(X, M, *)$ be a fuzzy metric space. Then, the upper proximal topology $\sigma_{M}^{++}$is the topology on $\mathcal{C}_{0}(X)$ which has as a base the sets of the form

$$
G^{++}=\left\{B \in \mathcal{C}_{0}(X): B_{M}(B, \varepsilon, t) \subseteq G \text { for some } \varepsilon \in(0,1) \text { and } t>0\right\},
$$

where $G$ is a nonempty $\tau_{M}$-open subset of $X$.

Remark 3.2. Let $(X, d)$ be a metric space. Then $\sigma_{d}^{++}=\sigma_{M_{d}}^{++}$on $\mathcal{C}_{0}(X)$.

Let us show this. Indeed, suppose that $A \in \mathcal{C}_{0}(X)$ and $B_{d}(A, \varepsilon) \subseteq G$ where $G$ is open. Let $0<t<1-\varepsilon$. We claim that $B_{M_{d}}(A, \varepsilon, t) \subseteq G$. In fact, suppose $M_{d}(a, y, t)>1-\varepsilon$ where $a \in A$. Hence, $d(a, y)<\varepsilon t /(1-\varepsilon)<\varepsilon$, so $y \in G$. In a similar way it can be proved that if $B_{M_{d}}(A, \varepsilon) \subseteq G$ then $B_{d}(A, \varepsilon t /(1-\varepsilon)) \subseteq G$.

Next we show that every upper proximal topology of a fuzzy metric space is a classical upper proximal topology. More precisely, we show that the upper proximal topology induced by a fuzzy metric space $(X, M, *)$ can be also induced by a certain metric $d$ on $X$.

Proposition 3.2. Let $(X, M, *)$ be a fuzzy metric space. Then $\sigma_{M}^{++}=\sigma_{d}^{++}$on $\mathcal{C}_{0}(X)$ where $d$ is any metric on $X$ generating the uniformity $\mathcal{U}_{M}$.

Proof. Suppose that $B_{M}(A, \varepsilon, t) \subseteq G$ where $A \in \mathcal{C}_{0}(X), \varepsilon \in(0,1), t>0$ and $G$ is a nonempty $\tau_{M}$-open subset of $X$. Since $d$ is compatible with $\mathcal{U}_{M}$ we can find $\delta>0$ such that

$$
\{(x, y) \in X \times X: d(x, y)<\delta\} \subseteq\{(x, y) \in X \times X: M(x, y, t)>1-\varepsilon\} .
$$

Consequently, $B_{d}(A, \delta) \subseteq B_{M}(A, \varepsilon, t) \subseteq G$.

Proceeding in a similar way, it is proved that if $B_{d}(A, \varepsilon) \subseteq G$ then $B_{M}(A, \delta, t) \subseteq G$ for some $\delta \in(0,1), t>0$.

The following result establishes the relationship between the upper Wijsman topology of a $C$-usc fuzzy metric space $(X, M, *)$ and its upper proximal topology.

Proposition 3.3. Let $(X, M, *)$ be a C-usc fuzzy metric space. Then $\tau_{W_{M}}^{+} \subseteq \sigma_{M}^{++}$.

Proof. Let $H=\left\{B \in \mathcal{C}_{0}(X): M(x, B, t)<1-\varepsilon\right\} \in \tau_{W_{M}}^{+}$where $x \in X, \varepsilon \in(0,1)$ and $t>0$.

If $H=\varnothing$, we obviously have $H \in \sigma_{M}^{++}$.

Then, suppose $H \neq \varnothing$ and let $A \in H$. Take $\delta>\varepsilon$ such that $M(x, A, t)<1-\delta$. Since $M(x, A, \cdot)$ is continuous at $t$ and $M(x, A, t)<1-\delta$ we can find an $s>t$ for 
which $M(x, A, s)<1-\delta$. Then, by continuity of $*$, there exists $\gamma \in(0,1)$ such that $M(x, A, s)<(1-\delta) *(1-\gamma)$.

Now choose $t^{\prime}>0$ such that $s>t^{\prime}>t$. We first notice that

$$
B_{M}\left(A, \gamma, s-t^{\prime}\right) \subseteq X \backslash \overline{B_{M}(x, \delta, t)} .
$$

Otherwise, we can find $y \in X, a \in A$ and a sequence $\left(z_{n}\right)_{n \in N}$ in $X$ such that $M(a, y, s-$ $\left.t^{\prime}\right)>1-\gamma, M\left(y, z_{n}, 1 / n\right)>1-1 / n$ and $M\left(x, z_{n}, t\right)>1-\delta$ for all $n \in \mathbb{N}$. Take $n_{0} \in \mathbb{N}$ such that $t^{\prime}>t+1 / n_{0}$. Then, for each $n \geq n_{0}$ we have

$$
\begin{aligned}
M(x, A, s) & \geq M(x, a, s) \geq M\left(x, z_{n}, t\right) * M\left(z_{n}, y, 1 / n\right) * M\left(y, a, s-t^{\prime}\right) \\
& \geq(1-\delta) *(1-1 / n) *(1-\gamma) .
\end{aligned}
$$

So, by continuity of $*, M(x, A, s) \geq(1-\delta) *(1-\gamma)$, which contradicts that $M(x, A, s)<$ $(1-\delta) *(1-\gamma)$. Furthermore, if $B \in\left(X \backslash \overline{B_{M}(x, \delta, t)}\right)^{++}$then $B \subseteq X \backslash \overline{B_{M}(x, \delta, t)}$ so, in particular, $B \cap B_{M}(x, \delta, t)=\varnothing$, i.e., $M(x, B, t) \leq 1-\delta<1-\varepsilon$, and thus $B \in H$. We conclude that $H \in \sigma_{M}^{++}$.

Example 3.1. We show that, in general, the above proposition is not true for an arbitrary fuzzy metric space. Let us consider the fuzzy metric space $\left(\mathbb{R}, M_{01}, \wedge\right)$ of Example 2.3 and the $\tau_{W_{M_{01}}}^{+}$-open set

$$
V=\left\{B \in \mathcal{C}_{0}(X): M_{01}\left(0, B, \frac{1}{2}\right)<\frac{1}{2}\right\} .
$$

It is obvious that $A=[1 / 2,1] \in V$.

On the other hand, it is easy to see that $\mathcal{U}_{M_{01}}=\mathcal{U}_{e}$, so , by Proposition 3.2, we deduce that $\sigma_{M_{01}}^{++}=\sigma_{e}^{++}$. Consequently, if $\tau_{W_{M_{01}}}^{+} \subseteq \sigma_{M_{01}}^{++}=\sigma_{e}^{++}$on $\mathcal{C}_{0}(X)$ then we could find an open set $G$ such that $A \in G^{++} \subseteq V$. Hence, there exists $\varepsilon>0$ with $B_{e}(A, \varepsilon) \subseteq G$, so $C=[(1-\varepsilon) / 2,1+\varepsilon / 2]$ also belongs to $G^{++}$. However, $M_{01}(0, C, 1 / 2)=1$, and thus $C \notin \in V$.

Notice that this example also shows that $\tau_{W_{M}}^{+}$is not weaker than $\tau_{V}^{+}$, in general.

Remark 3.3. It is well known $[1$, p. 50] that given two metrics $d, q$ on a nonempty set $X$, then $\sigma_{d}^{++}=\sigma_{q}^{++}$if and only if $\mathcal{U}_{d}=\mathcal{U}_{q}$. This is also true for fuzzy metrics. In fact, if $(M, *)$ and $(N, \star)$ are fuzzy metrics on $X$, then for any pair of metrics $d$ and $q$ on $X$ generating the uniformities $\mathcal{U}_{M}$ and $\mathcal{U}_{N}$, respectively, we deduce from Proposition 3.2 the following

$$
\sigma_{M}^{++}=\sigma_{N}^{++} \Leftrightarrow \sigma_{d}^{++}=\sigma_{q}^{++} \Leftrightarrow \mathcal{U}_{d}=\mathcal{U}_{q}=\mathcal{U}_{M}=\mathcal{U}_{N} .
$$

As promised, we now present two results which show that the Wijsman topology in our fuzzy context can be used to construct other hypertopologies [3, 1].

Proposition 3.4. Let $(X, M, *)$ be a $C$-usc fuzzy metric space and denote by $\mathcal{M}$ (resp. $\mathcal{D})$ the set of all $C$-usc fuzzy metrics (resp. metrics) on $X$ inducing the topology $\tau_{M}$. Then

$$
\sup \left\{\tau_{W_{N}}^{+}:(N, \star) \in \mathcal{M}\right\}=\sup \left\{\tau_{W_{d}}^{+}: d \in \mathcal{D}\right\}=\tau_{V}^{+} .
$$

Proof. The equality $\sup \left\{\tau_{W_{d}}^{+}: d \in \mathcal{D}\right\}=\tau_{V}^{+}$is well-known [3]. Now let $(N, \star) \in \mathcal{M}$. By Propositions 3.2 and 3.3 we have $\tau_{W_{N}}^{+} \subseteq \sigma_{N}^{++}=\sigma_{d}^{++}$for any metric $d$ generating the 
uniformity $\mathcal{U}_{N}$. Since $d \in \mathcal{D}$ and $\sigma_{d}^{++} \subseteq \tau_{V}^{+}$we deduce that $\sup \left\{\tau_{W_{N}}^{+}:(N, \star) \in \mathcal{M}\right\} \subseteq$ $\tau_{V}^{+}$.

Finally, given $d \in \mathcal{D}$, we have $\tau_{W_{d}}^{+}=\tau_{W_{M_{d}}}^{+}$(Example 2.1), so the other inclusion follows since $\left(M_{d}, *\right) \in \mathcal{M}$ for any continuous t-norm $*$.

Lemma 3.1 ([20, Proposition 2.5]). Let $(X, d)$ be a metric space. Then $\mathcal{U}_{d}=\mathcal{U}_{M_{d}}$.

Proposition 3.5. Let $(X, M, *)$ be a $C$-usc fuzzy metric space and denote by $\mathcal{M}$ (resp. $\mathcal{D})$ the set of all C-usc fuzzy metrics (resp. metrics) on $X$ inducing the topology $\tau_{M}$. Then $\sup \left\{\tau_{W_{N}}^{+}:(N, \star) \in \mathcal{M}\right.$ and $\left.\mathcal{U}_{N}=\mathcal{U}_{M}\right\}=\sup \left\{\tau_{W_{q}}^{+}: q \in \mathcal{D}\right.$ and $\left.\mathcal{U}_{q}=\mathcal{U}_{M}\right\}=\sigma_{M}^{++}$.

Proof. Let $d \in \mathcal{D}$ with $\mathcal{U}_{d}=\mathcal{U}_{M}$. It is known [3] that $\sup \left\{\tau_{W_{q}}^{+}: q \in \mathcal{D}\right.$ and $\mathcal{U}_{q}=$ $\left.\mathcal{U}_{M}\right\}=\sigma_{d}^{++}$and by Proposition 3.2 this topology equals to $\sigma_{M}^{++}$. Now let $(N, \star) \in \mathcal{M}$ such that $\mathcal{U}_{N}=\mathcal{U}_{M}$. By Proposition 3.3 and Remark 3.3 we have $\tau_{W_{N}}^{+} \subseteq \sigma_{M}^{++}$. We deduce that $\sup \left\{\tau_{W_{N}}^{+}:(N, \star) \in \mathcal{M}\right.$ and $\left.\mathcal{U}_{N}=\mathcal{U}_{M}\right\} \subseteq \sigma_{M}^{++}$.

Finally, given $q \in \mathcal{D}$ such that $\mathcal{U}_{q}=\mathcal{U}_{M}$, we have, by Lemma 3.1, $\mathcal{U}_{M_{q}}=\mathcal{U}_{M}$. Consequently $\tau_{W_{q}}^{+}=\tau_{W_{M_{q}}}^{+} \subseteq \sup \left\{\tau_{W_{N}}^{+}:(N, \star) \in \mathcal{M}\right.$ and $\left.\mathcal{U}_{N}=\mathcal{U}_{M}\right\}$.

In view of Proposition 3.2 it is natural to wonder if given a fuzzy metric space $(X, M, *)$, we can find a metric $d$ on $X$ such that $\tau_{W_{M}}=\tau_{W_{d}}$. It seems that a suitable candidate for $d$ should be a metric compatible with $\mathcal{U}_{M}$. We conclude the paper with an example showing that not every metric with this property satisfies the assertion.

Example 3.2. Let $d_{0}$ be the discrete metric on $\mathbb{N}$ and let $d_{1}$ be the metric on $\mathbb{N}$ given by $d_{1}(n, n)=0$ for all $n \in \mathbb{N}, d_{1}(1, n)=2$ for all $n>1$, and $d_{1}(n, m)=1$ for all $n, m>1$ with $n \neq m$. Then $\mathcal{U}_{d_{0}}=\mathcal{U}_{d_{1}}$, so by Lemma 3.1, $\mathcal{U}_{M_{d_{0}}}=\mathcal{U}_{d_{1}}$. However $\tau_{W_{d_{0}}} \neq \tau_{W_{d_{1}}}[1$, Example 2.1.3 and p. 38], and hence (see Example 2.1), $\tau_{W_{M_{d_{0}}}} \neq \tau_{W_{M_{d_{1}}}}$.

\section{Conclusion and further work}

We have introduced and studied a notion for the Wijsman topology induced by a fuzzy metric space in the sense of Kramosil and Michalek. Although our approach seems to be the natural one, the situation presents some differences with respect to the classical case of metric spaces as the Wijsman topology is not admissible. Nevertheless, we have been able to obtain fundamental result about this topology. In particular, we have characterized metrizability of the Wijsman topology of a fuzzy metric and we have emphasized that our results allow to improve classical results about hypertopologies.

In a further work, we will try to generalize, if possible, our results to any fuzzy metric space $(X, M, *)$, deleting the $C$-usc condition. This would entail to look for a different definition of the upper Wijsman topology in the fuzzy context in such a way that the Wijsman topology be admissible and have a good behaviour in the hypertopological lattice. 


\section{Acknowledgements}

J. Gutiérrez García acknowledges the support of the Ministry of Economy and Competitiveness of Spain, Grant MTM2012-37894-C02-02. J. Rodríguez-López, S. Romaguera and M. Sanchis also acknowledge the support of the Ministry of Economy and Competitiveness of Spain, Grant MTM2012-37894-C02-01.

\section{References}

[1] G. Beer, Topologies on Closed and Closed Convex Sets, Vol. 268, Kluwer Acad. Publ., 1993.

[2] G. Beer, Wijsman convergence: A survey, Set-Valued Anal. 2 (1994), 77-94.

[3] G. Beer, A. Lechicki, S. Levi, S. Naimpally, Distance functionals and suprema of hyperspace topologies, Ann. Mat. Pura Appl. 162 (1992), 367-381.

[4] G. Berthiaume, On quasi-uniformities in hyperspaces, Proc. Amer. Math. Soc. 66 (1977) 335-343.

[5] J. Cao, H.J.K. Junnila, Hereditarily normal Wijsman hyperspaces are metrizable, Top. Appl. 169 (2014), 148-155.

[6] J. Cao, H.J.K. Junnila, W.B. Moors, Wijsman hyperspaces: Subspaces and embeddings, Topology and its Applications 159 (2012), 1620-1624.

[7] F. Castro-Company, S. Romaguera, P. Tirado, The bicompletion of fuzzy quasimetric spaces, Fuzzy Sets Syst. 166 (2011), 56-64.

[8] Y.J. Cho, M. Grabiec, V. Radu, On Nonsymmetric Topological and Probabilistic Structures, Nova Sci. Publ., Inc. New York, 2006.

[9] S. Cobzaş, Functional Analysis in Asymmetric Normed Spaces, Birkhäuser, Springer Basel, 2013.

[10] C. Costantini, Every Wijsman topology relative to a Polish space is Polish, Proc. Amer. Math. Soc. 123 (1995), 2569-2574.

[11] C. Costantini, S. Levi, J. Pelant, Infima of hyperspace topologies, Mathematica 42 (1995), 67-86.

[12] G. Di Maio, Ĺ. Holá, Wijsman topology on function spaces, Rend. Cir. Mat. Palermo, Ser. II 46 (1997), 52-70.

[13] R.J. Egbert, Products and quotients of probabilistic metric spaces, Pacific J. Math. 24 (1968), 437-455.

[14] R. Engelking, General Topology, Heldermann Verlag, Berlin, 1989.

[15] P. Fletcher, W.F. Lindgren, Quasi-Uniform Spaces, Marcel Dekker, New York, 1982. 
[16] A. George, P. Veeramani, On some results in fuzzy metric spaces, Fuzzy Sets Syst. 64 (1994), 395-399.

[17] V. Gregori, S. Romaguera, Some properties of fuzzy metric spaces, Fuzzy Sets Syst. 115 (2000), 485-489.

[18] V. Gregori, S. Romaguera, Characterizing completable fuzzy metric spaces, Fuzzy Sets Syst. 144 (2004), 411-420.

[19] V. Gregori, S. Romaguera, Fuzzy quasi-metric spaces, Appl. Gen. Topology 5 (2004), 129-136.

[20] J. Gutiérrez García, S. Romaguera, M. Sanchis, Fuzzy uniform structures and continuous t-norms, Fuzzy Sets Syst. 161 (2010), 1011-1021.

[21] J. Gutiérrez García, S. Romaguera, M. Sanchis, An identification theorem for the completion of the Hausdorff fuzzy metric, Fuzzy Sets Syst. 227 (2013), 96-106.

[22] E.P. Klement, R. Mesiar, E. Pap, Triangular Norms, Kluwer Acad. Publ., Dordrecht, 2000.

[23] I. Kramosil, J. Michalek, Fuzzy metrics and statistical metric spaces, Kybernetika 11 (1975), 326-334.

[24] H.P.A. Künzi, C. Ryser, The Bourbaki quasi-uniformity, Topology Proc. 20 (1995) 161-183.

[25] J. Rodríguez-López, S. Romaguera, Wijsman and hit-and-miss topologies of quasimetric spaces, Set-Valued Anal. 11 (2003) 323-344.

[26] J. Rodríguez-López, S.Romaguera, J.M. Sánchez-Álvarez, The Hausdorff fuzzy quasi-metric, Fuzzy Sets Syst. 161 (2010), 1078-1096.

[27] B. Schweizer, A. Sklar, Statistical metric spaces, Pacific J. Math. 10 (1960), 314334.

[28] C.Sempi, Hausdorff distance and the completion of probabilistic metric spaces, Boll. Un. Mat. Ital. 6-B (1992), 317-327.

[29] R.M. Tardiff, Topologies for probabilistic metric spaces, Pacific J. Math. 65 (1976), 233-251.

[30] R.A. Wijsman, Convergence of sequences of convex sets, cones and functions. II, Trans. Amer. Math. Soc. 123 (1966), 32-45.

[31] L. Zsilinszky, Polishness of the Wijsman topology revisited, Proc. Amer. Math. Soc. 126 (1998), 3763-3765. 